\title{
The effect of cyclodextrin-solubilized curcuminoids on amyloid plaques in Alzheimer transgenic mice: brain uptake and metabolism after intravenous and subcutaneous injection
}

\author{
Wolfgang W Quitschke ${ }^{1 *}$, Nicole Steinhauff ${ }^{2}$ and Jean Rooney ${ }^{2}$
}

\begin{abstract}
Introduction: Curcuminoids may improve pathological conditions associated with Alzheimer's disease. However, their therapeutic potential is limited by their exceedingly low bioavailability after oral administration. A method to deliver solubilized curcuminoids by injection was evaluated in Alzheimer transgenic mice.

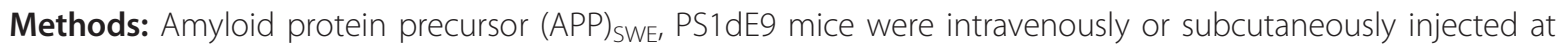
weekly intervals between the ages of 4 and 12 months with serum- or cyclodextrin-solubilized curcuminoids to assess their potential for plaque prevention. Alternatively, mice between the ages of 11 and 12 months were intravenously injected with cyclodextrin-solubilized curcuminoids at biweekly intervals to evaluate their ability to eliminate existing plaques. Plasma and brain levels of curcuminoids and their metabolites were also determined after subcutaneous and intravenous injection.

Results: Weekly long-term injections did not result in a significant plaque load reduction. However, intravenous injection of cyclodextrin-solubilized curcuminoids at higher curcuminoid concentrations and at a biweekly frequency between the ages of 11 and 12 months reduced the plaque load to approximately $70 \%$ of the control value. After intravenous injection, plasma levels of $100 \mu \mathrm{M}$ curcuminoids and brain levels of $47 \mathrm{nmol} / \mathrm{g}$ could initially be achieved that declined to essentially undetectable levels within 20 minutes. The primary curcuminoid metabolites in plasma were the conjugates of glucuronide or sulfate and hexahydrocurcuminoids as reduction products. In the brain, both hexahydrocurcuminoids and octahydrocurcuminoids were detected as major metabolites. After subcutaneous injection, maximal curcuminoid plasma levels of $23 \mu \mathrm{M}$ and brain levels of 8 $\mathrm{nmol} / \mathrm{g}$ were observed at 30 minutes after injection and curcuminoids remained detectable for 2 to $3 \mathrm{~h}$.
\end{abstract}

Conclusion: Curcuminoids are rapidly metabolized after injection and their effect on reducing plaque load associated with Alzheimer's disease may be dependent on the frequency of administration.

\section{Introduction}

Curcumin is a yellow pigment extracted from the spice and coloring agent turmeric, where it occurs in amounts of 2 to $8 \%$ [1]. Commercial curcumin preparations typically contain a mixture of polyphenols, collectively referred to as curcuminoids. In addition to the primary component curcumin (CUR, 65 to $80 \%$ ), they also

\footnotetext{
* Correspondence: wolfgang.quitschke@stonybrook.edu

'Department of Psychiatry and Behavioral Science, Stony Brook University Medical Center, 100 Nicolls Road, Stony Brook, New York 11794-8101, USA Full list of author information is available at the end of the article
}

contain smaller amounts of the co-extracted congeners demethoxycurcumin (DMC, 15 to $25 \%$ ) and bisdemethoxycurcumin (BDMC, 5 to $15 \%$ ) [2-4].

Curcumin binds to $A \beta$ fibrils, presumably in the enol form [5], and stains amyloid plaques and neurofibrillary tangles in brain sections [6,7] and in vivo [8-10]. Curcumin inhibits $A \beta$ fibril formation and promotes disaggregation of existing fibrils in vitro with $\mathrm{IC}_{50}$ values of 0.19 to $1 \mu \mathrm{M}[9,11,12]$, although much higher $\mathrm{IC}_{50}$ values in the 10 to $12 \mu \mathrm{M}$ range have been reported [13,14]. Curcumin similarly inhibits plaque formation or disrupts

\section{C) Biomed Central}


existing plaques in Alzheimer transgenic mouse models either after oral uptake [9,15-17] or intravenous (i.v.) injection [8]. Apart from the direct interaction with $A \beta$ fibrils, curcuminoids may reduce plaque formation or ameliorate their effects by increasing $A \beta$ uptake by macrophages [18], affecting amyloid protein precursor (APP) maturation [19], modulating APP processing enzymes $[20,21]$, protecting neurons from $A \beta$ induced toxicity $[9,22-24]$ or influencing the expression of genes associated with apoptosis and inflammation [25]. Alternatively, curcumin degradation products may mediate similar effects (Review: [26]).

Despite such promising observations, the clinical use of orally administered curcuminoids is severely limited by their exceedingly low bioavailability, which is a direct consequence of their poor solubility in aqueous solutions and their rapid metabolic conversion (Reviews: [26-28]). To improve systemic availability, formulations containing high concentrations of curcuminoids were solubilized in either serum [29] or 2-hydroxypropyl- $\gamma$ cyclodextrin (HP- $\gamma-\mathrm{CD})$ and injected into Alzheimer transgenic mice. The effect on plaque development, systemic availability and metabolism was investigated.

\section{Materials and methods}

\section{Curcuminoid solubilization}

Technical grade curcumin (Cayman Chemical Company, Ann Arbor, MI, USA) containing CUR (69\%), DMC (19\%) and BDMC (12\%) was solubilized in either C57BL/6 mouse serum (Valley Biomedical, Winchester, VA, USA) or in an aqueous solution of $10 \% \mathrm{HP}-\gamma-\mathrm{CD}$ (Sigma-Aldrich, St. Louis, MO, USA) containing 0.6\% $\mathrm{NaCl}, \mathrm{pH} 6.8$ by the sequential mixing with solid- and DMSO-dissolved curcumin as described elsewhere [29]. Briefly, $50 \mathrm{ml}$ of serum or $10 \% \mathrm{HP}-\gamma-\mathrm{CD}$ was mixed by stirring with solid curcuminoids $(50 \mathrm{mg} / \mathrm{ml})$ for $16 \mathrm{~h}$ at $4^{\circ} \mathrm{C}$. Thereafter, the suspension was clarified by centrifugation at $18,000 \times \mathrm{g}$ for 30 minutes. The supernatant was removed and DMSO-dissolved curcuminoids at a 1 $M$ concentration were added $(10 \mu \mathrm{l} / \mathrm{ml})$ and again stirred for $16 \mathrm{~h}$. The resulting suspension was then clarified by two successive centrifugations at $18,000 \times \mathrm{g}$. The supernatant was sterilized by filtration through a 0.45 $\mu \mathrm{m}$ membrane filter (Pall Corporation, Ann Arbor, MI, USA). The final supernatants contained either 3-4 mM total curcuminoids solubilized in serum or $24 \mathrm{mM}$ curcuminoids solubilized in 10\% HP- $\gamma$-CD (full-strength).

\section{Animals, injections and tissue preparation}

For the study on plaque prevention, female APP $\mathrm{SWE}_{\mathrm{E}}$, PS1dE9 transgenic mice (Jackson Laboratories, Bar Harbor, ME, USA) were i.v. injected with serum-solubilized curcumin via the tail vein once per week starting at four months of age. The average weight of the mice was about $25 \mathrm{~g}$ (range: 23 to $27 \mathrm{~g}$ ) and they were injected with a total volume of $0.1 \mathrm{ml}$ serum-solubilized curcuminoids. This protocol was discontinued after five injections (four weeks) due to adverse reactions. As an alternative, full-strength HP- $\gamma$-CD-solubilized curcuminoids were diluted four-fold with isotonic saline to a concentration of $6 \mathrm{mM}$ and similarly injected until the mice had reached the age of about 10.5 months. For the remaining six weeks, the mice were subcutaneously (s.c.) injected between the shoulder blades at weekly intervals with $0.4 \mathrm{ml}$ full-strength HP- $\gamma$-CD-solubilized curcuminoids until the final age of 12 months. For the study on plaque resolution, mice at 11 months of age were i.v. injected for 1 month twice weekly (nine injections) with $0.1 \mathrm{ml}$ of full-strength HP- $\gamma$-CD-solubilized curcuminoids. The brains from a total of 23 mice were successfully processed for histological evaluation. These included one negative control without transgenes, three positive controls without injections, three positive controls each with injections for plaque prevention and plaque resolution, six experimental mice injected for plaque prevention and seven mice for plaque resolution.

Brain and plasma levels following curcuminoid injection were analyzed in wild type $\mathrm{C} 57 \mathrm{BL} / \mathrm{CH} 3$, transgenic $\mathrm{C} 57 \mathrm{BL} / \mathrm{CH} 3$ and $\mathrm{CV}-1$ mice. Since the $\mathrm{CV}-1$ mice were larger with an average weight of about $50 \mathrm{~g}$ (range: 45 to $55 \mathrm{~g}$ ), these were i.v injected with $0.2 \mathrm{ml}$ or s.c. injected with $0.8 \mathrm{ml}$ of full-strength HP- $\gamma$-CD-solubilized curcuminoids to account for the larger mass and blood volume. After injection, mice were sacrificed by exposure to $\mathrm{CO}_{2}$ at selected time intervals. Blood (approximately 1 $\mathrm{ml}$ ) was collected by cardiac puncture into tubes containing EDTA and the tissues were subsequently removed and stored at $-75^{\circ} \mathrm{C}$ until further processing. The blood was centrifuged at $14,000 \times \mathrm{g}$ for one minute for plasma collection.

To estimate the maximum uptake/binding of curcuminoids in the brain under the applied i.v. injection conditions, one CV-1 mouse was exposed to curcuminoids by cardiac perfusion, as modified from the procedure described below. The mouse was sequentially perfused with $10 \mathrm{ml}$ mouse serum, $30 \mathrm{ml}$ mouse serum containing $5 \%$ full-strength $\mathrm{HP}-\gamma$-CD-solubilized curcuminoids, and then washed with $30 \mathrm{ml}$ of PBS.

All procedures involving animal handling and processing were approved by the Stony Brook University Institutional Animal Care and Use Committee (IACUC) in compliance with the National Institutes of Health guidelines.

\section{Amyloid plaque staining of brain sections and quantitative analysis}

At the age of 12 months, all mice were prepared for histological evaluation. Terminal deep anesthesia was 
induced by s.c. injection of $0.2 \mathrm{ml}$ of pentobarbital $(50 \mathrm{mg} / \mathrm{ml})$. The chest cavity was exposed and a blunt syringe needle inserted into the left ventricle of the heart. The right atrium was cut open and the mice were sequentially perfused with $10 \mathrm{ml}$ of wash solution ( $\mathrm{NaCl}, 8 \mathrm{~g} / \mathrm{L}$; dextrose, $4 \mathrm{~g} / \mathrm{L}$; anhydrous $\mathrm{CaCl}_{2}, 0.23 \mathrm{~g} /$ $\mathrm{L}$; sodium cacodylate trihydrate, $0.34 \mathrm{~g} / \mathrm{L}$ ) and $30 \mathrm{ml}$ of fix solution (sucrose, $40 \mathrm{~g} / \mathrm{L}$; paraformaldehyde, $40 \mathrm{~g} / \mathrm{L}$; sodium cacodylate, $14.34 \mathrm{~g} / \mathrm{L} ; \mathrm{pH} 7.2$ ). The whole heads stripped of skin and eyes were placed in $30 \mathrm{ml}$ of sodium cacodylate buffer $(0.2 \mathrm{M}, \mathrm{pH} 7.4)$ and shipped to NeuroScience Associates (Knoxville, TN, USA) for further processing.

Brains were removed from skulls and treated with $20 \%$ glycerol and 2\% dimethylsulfoxide to prevent freezeartifacts, and embedded in a gelatin matrix using MultiBrain Technology ${ }^{\circledR}$ (NeuroScience Associates). After curing, the block of embedded brains was rapidly frozen by immersion in isopentane chilled to $-70^{\circ} \mathrm{C}$ with crushed dry ice, and mounted on a freezing stage of an AO 860 sliding microtome. The MultiBrain ${ }^{\mathbb{B}}$ block was sectioned coronally at $35 \mu \mathrm{m}$. Sections were sequentially collected into 24 containers that were filled with Antigen Preserve solution (49\% PBS pH 7.0, 50\% ethylene glycol, 1\% polyvinyl pyrrolidone). At the completion of sectioning, each container held a serial set of 1-of-every-24th section (one section every $840 \mu \mathrm{m})$. Each of the MultiBrain ${ }^{\mathbb{B}}$ sections cut from the block was a composite holding individual sections from each of the brains embedded in the block. With such composite sections, uniformity of staining was achieved across treatment groups.

Brain sections were silver-stained according to the Campbell-Switzer Alzheimer's method for plaques and tangles [30,31]. Overall, this stain displays sensitivity and specificity properties comparable to immunohistochemical methods [32-35]. MultiBrain ${ }^{\circledR}$ sections were collected and stained free-floating at room temperature. The sections were placed in freshly prepared $2 \%$ ammonium hydroxide for five minutes, and then transferred sequentially to a silver-pyridine-carbonate solution for 40 minutes, $1 \%$ citric acid for 3 minutes and $0.5 \%$ acetic acid until ready for development. The sections were developed in Physical Developer ABC solution (after Gallyas; containing sodium carbonate, citric acid, tungstosilicic acid and formaldehyde) with the development time being visually assessed. The development was stopped by placing the sections briefly in $0.5 \%$ acetic acid. Sections were mounted on gelatinized (subbed) glass slides, dehydrated and cover-slipped.

The Alzheimer's disease (AD) plaque burden was determined by quantifying and averaging the plaque area percentage of cortical area from four sections per animal. The coronal section containing the full anterior commissure was chosen as the first section to quantify, and the subsequent three stained sections completed the set of four. Individual slides containing an entire MultiBrain ${ }^{\circledR}$ section were scanned at $4 \mathrm{X}$ as high-resolution 24 bit color tif files. The color density of the images was adjusted in Photoshop ${ }^{\circledR}$ to provide contrast between the plaques and the background, but without using Brightness-Contrast as this function changes the size of the plaques. The threshold of each image was determined to provide an image with plaques and no background in Image $(\mathrm{NIH}$, Bethesda, MD, USA). Using the threshold adjustment, a level was determined that removed the background while leaving plaques intact. Plaque size and number was quantified in Image J with Size $=0$ to infinity and circularity $=0$ to 1 . From Analyze > Particles, the result was copied into a spreadsheet for the appropriate level of the image ( 1 to 4). This process was repeated for all images. Percent plaque area was calculated in Excel ${ }^{\circledR}$ and graphed in SigmaPlot $^{\mathrm{TM}}$ (11.2) according to animal number and plaque density.

\section{Tissue and plasma extraction for reversed phase chromatography}

Approximately $200 \mathrm{mg}$ of brain tissue (approximately one hemisphere) was homogenized in $0.7 \mathrm{ml}$ of $50 \%$ acetonitrile, $0.01 \%$ ammonium acetate, $\mathrm{pH} 4.5$ (total volume approximately $0.9 \mathrm{ml}$ ). The homogenate was transferred to a $1.5 \mathrm{ml}$ microcentrifuge tube and centrifuged for five minutes at $18,000 \times$ g. Subsequently, 0.4 $\mathrm{ml}$ of the supernatant was removed and transferred to a fresh microcentrifuge tube, which was then mixed with $0.8 \mathrm{ml}$ of acetone and left for 12 to $16 \mathrm{~h}$ at $-20^{\circ} \mathrm{C}$. The precipitated protein was centrifuged at $14,000 \times \mathrm{g}$ for five minutes and the clear supernatant was transferred to a fresh tube and evaporated under vacuum. The dry residue was re-solubilized in $0.2 \mathrm{ml} 37.5 \%$ acetonitrile, $0.01 \%$ ammonium acetate, $\mathrm{pH} 4.5$ and clarified by centrifugation and the supernatant was loaded onto a reversed phase column and separated as described elsewhere $[29,36]$. Plasma $(0.2 \mathrm{ml})$ was mixed with an equal volume of $50 \%$ acetonitrile, acetone precipitated and then processed the same way as the brain tissue.

\section{Analysis of curcuminoids and metabolites}

Curcuminoids and their conjugated metabolites were separated by reversed phase chromatography and monitored at an absorption wavelength of $427 \mathrm{~nm}$. Curcuminoids were quantitated based on standard curves for curcumin (analytical grade) by integration of the peaks with the FPLC Unicorn $^{\mathrm{TM}}$ (version 5.10) program (GE Healthcare Life Sciences, Piscataway, NJ, USA). The molar absorptivities $(\varepsilon)$ of the three curcuminoids dissolved in ethanol were shown to range from $6.73\left(\mathrm{x} 10^{4} \mathrm{~L} \mathrm{~cm}^{-1} \mathrm{~mol}^{-1}\right)$ for CUR, 5.78 for DMC and 4.95 for BDMC at $425 \mathrm{~nm}$ [37]. Since the molar absorptivity for curcumin dissolved in 
acetonitrile was essentially the same as in ethanol, the integrated values of the DMC and BDMC peaks were multiplied by the respective factors 1.17 and 1.36 to obtain molar concentrations in all experiments. The conjugated metabolites were identified by mass spectrometry as described elsewhere [36] and they were assumed to have the same molar absorption as their parental counterparts [38]. The reductive metabolites of curcuminoids were visualized at an absorption wavelength of $280 \mathrm{~nm}$ or 310 $\mathrm{nm}$. The molar absorptivities of the hexa- and octahydrocurcuminoids were taken from the data presented by Hoehle et al. [39], which were in excellent agreement with standard curves generated with commercial preparations of hexahydrocurcumin (Sigma-Aldrich) and octahydrocurcumin (Sabinsa Corporation, East Windsor, NJ, USA). Unless otherwise indicated, all data points were calculated as the average of 3 to 10 independent experiments. Error bars represent the standard deviation from the mean.

\section{Curcuminoid binding to NT2/D1 cells}

NT2/D1 cells were grown in $25 \mathrm{~cm}^{2}$ flasks to a density of about $90 \%$ confluence $\left(6\right.$ to $8 \times 10^{6}$ cells) with $6 \mathrm{ml}$ of Dulbecco's Minimal Essential Medium (DMEM) containing 5\% fetal calf serum (FCS). For curcuminoid dose curves to determine binding dissociation constants, media were prepared with increasing amounts $(10 \mu \mathrm{l}$ to 2 $\mathrm{ml}$ ) of curcuminoids solubilized in mouse serum or $10 \%$ HP- $\gamma-C D$ and supplemented with DMEM for a total volume of $6 \mathrm{ml}$. Media for serum competition curves were prepared by adding $300 \mu \mathrm{l}$ of solubilized curcuminoids to a total volume of $6 \mathrm{ml}$. The remaining volume consisted of varying ratios of DMEM and mouse serum to generate dose curves with constant curcuminoid and variable serum concentrations. Curcuminoids solubilized in mouse serum at a $4 \mathrm{mM}$ initial concentration yielded a final media concentration of $200 \mu \mathrm{M}$. Curcuminoids solubilized in HP- $\gamma$-CD were either used full-strength (24 $\mathrm{mM})$ or diluted with $0.6 \%$ saline to a $6 \mathrm{mM}$ concentration, resulting in final media concentrations of $1.2 \mathrm{mM}$ and $300 \mu \mathrm{M}$, respectively. After $1 \mathrm{~h}$ incubation, $0.4 \mathrm{ml}$ of medium was withdrawn for the determination of free curcuminoids and mixed with $0.8 \mathrm{ml}$ of acetone. Cells were then washed three times in the flasks with $5 \mathrm{~mL}$ of $0.9 \%$ saline and then scraped into $1 \mathrm{ml}$ of the same solution. The cell suspension was centrifuged at $3,000 \times \mathrm{g}$ for one minute and the supernatant removed. The cell pellet was resuspended in $1 \mathrm{ml}$ of $0.6 \%$ saline and re-centrifuged. The final pellet was suspended in $0.4 \mathrm{ml}$ of $0.6 \%$ saline and mixed with $0.8 \mathrm{ml}$ of acetone. All samples were stored at $-20^{\circ} \mathrm{C}$ for 2 to $16 \mathrm{~h}$ and processed for reversed phase chromatography as described above for tissue preparation. After curcuminoid quantitation, data points were combined from three independent experiments, normalized to $10^{6}$ cells, and fitted to a ligand binding function $\left(\mathrm{y}=\left(\mathrm{B}_{\mathrm{MAX}}{ }^{*} \mathrm{x}\right) /\left(\mathrm{K}_{\mathrm{D}}+\mathrm{x}\right)\right.$ $\left.+\mathrm{N}_{\mathrm{S}} \mathrm{x}\right)$ or a hyperbolic decay function $(\mathrm{y}=\mathrm{ab} /(\mathrm{b}+\mathrm{x}))$ using SigmaPlot ${ }^{\mathrm{TM}}(11.2)$ as described elsewhere [36].

\section{Results}

\section{Curcuminoids solubilized in serum and HP- $\gamma$-CD}

To assess the use of solubilized curcuminoids as a therapeutic or preventative agent in the treatment of Alzheimer's disease, technical grade curcumin was initially solubilized in mouse serum. Technical grade curcumin is the type of preparation used in the vast majority of studies on this compound and it contains all three curcuminoids as they are represented in the turmeric powder. All three curcuminoids were included in this study to neutralize any effects due to their differential chemical stability, metabolic conversion or cellular binding affinity $[29,36]$. Furthermore, all three curcuminoids have been shown to inhibit $A \beta$ fibrillogenesis and they may complement each other to inhibit the process that leads to plaque formation [40]. The sequential solubilization of solid and DMSO-dissolved curcumin yielded a total soluble concentration of 3-4 $\mathrm{mM}$ and a curcuminoid distribution as depicted in Figure 1A. The feasibility of using such serum-solubilized curcuminoids for i.v. injection was initially tested on Sprague-Dawley rats using serum derived from random animals of the same strain. Rats were injected weekly via the tail vein with serum-solubilized curcuminoids at approximately $7 \%$ of the total blood volume for up to four months without adverse effects.

A similar injection protocol was adapted for the Alzheimer transgenic mouse strain $\mathrm{APP}_{\mathrm{SWE}}$, PS1dE9, which is a hybrid from a cross between $\mathrm{C}_{57} \mathrm{BL} / 6$ and $\mathrm{C}_{3} \mathrm{H}$ mice [41]. Since serum from such a cross is commercially unavailable, serum derived from $\mathrm{C}_{57} \mathrm{BL} / 6$ mice was used. The first two injections proceeded uneventfully. However, after the third injection adverse reactions occurred that were consistent with anaphylaxis. Since such reactions were also observed in control mice injected with serum devoid of curcuminoids, it was concluded that these were caused by incompatible serum components. Most mice recovered within one hour; however, some fatalities occurred. It was, therefore, deemed impossible to continue with such serum injections and a new vehicle for the solubilization of injectable curcumin had to be found.

Solubilizing curcuminoids in $10 \% \mathrm{HP}-\gamma-\mathrm{CD}$ proved to be ideal for this purpose. Mixing either solid curcuminoids or DMSO-dissolved curcuminoids with $10 \% \mathrm{HP}-\gamma$ $\mathrm{CD}$, preferentially solubilized BDMC or CUR, respectively. As such, the distribution of individual curcuminoids followed the same pattern as that observed with serum or albumin solutions [29]. To maximize the amount of curcuminoids solubilized and to limit the 


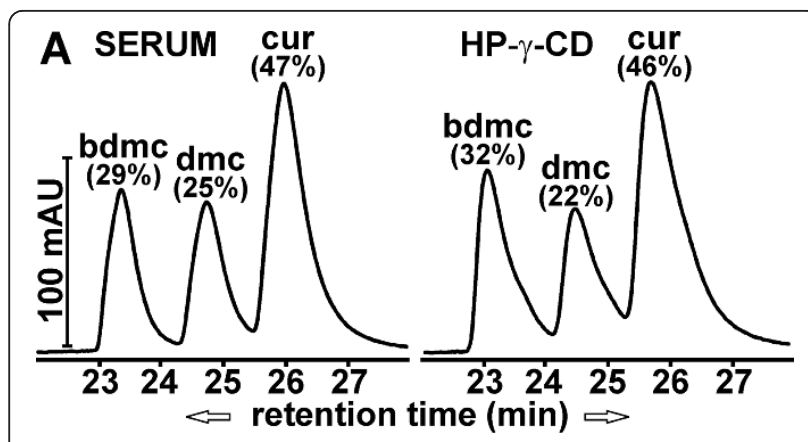

B
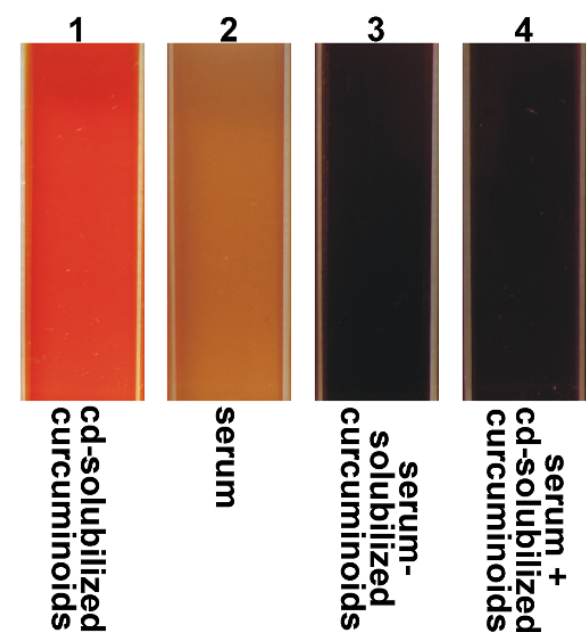

Figure 1 Curcuminoid solubilization. (A) Chromatograms of curcuminoids solubilized either in serum (left panel) or $10 \% \mathrm{HP}-\gamma$ CD (right panel). The relative contribution of each curcuminoid (\%) relative to the total amount (100\%) is indicated. (B) The color of curcuminoids solublized in 10\% HP- $\gamma$-CD (1) compared to normal serum (2), curcuminoids solubilized directly in serum (3), and HP- $\gamma$ CD-solubililized curcuminoids diluted to $5 \%$ with serum (4).

final concentration of DMSO to $1 \%$, curcuminoids were added sequentially, first as a solid powder followed by DMSO-dissolved curcuminoids. This resulted in a curcuminoid distribution that was very similar to that obtained with serum by the same procedure (Figure $1 \mathrm{~A})$, albeit with a higher final curcuminoid concentration of approximately $24 \mathrm{mM}$. Upon diluting such curcuminoid solutions with serum, no precipitation occurred that might have had adverse effects on the circulation. In addition, the HP- $\gamma$-CD solubilized curcuminoids partially equilibrated with serum components as evidenced by a shift in color from yellow to deep red, which is similar to the color of curcuminoids directly solubilized in serum (Figure 1B). For long-term i.v. injection studies, the full-strength HP- $\gamma$-CD-solubilized curcuminoids were diluted four-fold to a final concentration of $6 \mathrm{mM}$ curcuminoids in $2.5 \% \mathrm{HP}-\gamma$-CD. This resulted in a curcuminoid concentration similar to that obtained by serum-solubilization, which caused no adverse effects. In addition, the amount of HP- $\gamma-C D$ $(100 \mathrm{mg} / \mathrm{kg})$ injected was below that considered toxic for chronic exposure [42].

\section{The effect of injecting soluble curcuminoids on plaques} in Alzheimer transgenic mice

The transgenic mouse line $\mathrm{APP}_{\mathrm{SWE}}$, PS1dE9 contains the Swedish mutations (K595N/M596L) within a chimeric mouse/human APP695, and the human transgene for presenilin 1 with a deletion of exon 9 [41]. This mouse line begins to develop plaques at 4 to 6 months of age, which progresses to extensive plaque load by 12 months of age [43]. The ability of curcuminoids to prevent amyloid plaque formation was studied by weekly tail vein injections. Injections of $0.1 \mathrm{ml}$ serum-solubilized curcuminoids $(4 \mathrm{mM})$ were started at the age of four months and replaced with HP- $\gamma$-CD-solubilized curcuminoids (6 $\mathrm{mM}$ ) four weeks later. At the age of about 10.5 months, the tail veins of many mice had deteriorated to the point that further i.v. injections were rendered impossible. For the last six weeks, until the age of 12 months, tail vein injections were, therefore, replaced with weekly s.c. injections of $0.4 \mathrm{ml}$ full-strength $(24 \mathrm{mM}) \mathrm{HP}-\gamma$-CDsolubilized curcuminoids. The ability of curcuminoids to resolve existing plaques was investigated by tail vein injection twice weekly with $0.1 \mathrm{ml}$ of $24 \mathrm{mM} \mathrm{HP}-\gamma-\mathrm{CD}-$ solubilized curcuminoids. Nine injections were carried out between the ages of 11 and 12 months (Figure 2A).

The i.v. injection of $6 \mathrm{mM} \mathrm{HP}-\gamma$-CD-solubilized curcuminoids caused no obvious detrimental effects. However, within seconds after i.v. injection of curcuminoids at $24 \mathrm{mM}$ concentrations, acute reactions were observed that included trembling and agitation followed by a period of lethargy. These reactions subsided within five minutes after injection and caused no deaths in otherwise healthy animals. Such effects were not observed in control animals and it was concluded that curcuminoids were toxic at these high concentrations. The rapid resolution of symptoms is consistent with the notion that curcuminoids are rapidly metabolized. No adverse effects were noted after s.c. injections.

At the age of 12 months, transgenic mice showed vigorous amyloid plaque development compared to a negative control not carrying the transgene (Figure 2B, $C)$. The plaque load in the experimental groups was determined for plaque prevention $(N=6)$ and for plaque resolution $(N=7)$. Included were also positive control mice that had not received any injections $(N=3)$, and mice from each group $(N=3)$ that had received injections of the solubilization vehicle without curcuminoids. The average plaque loads between the three control groups showed no significant differences. For statistical purposes, the three control groups were, therefore, combined $(N=9)$ and compared with the 

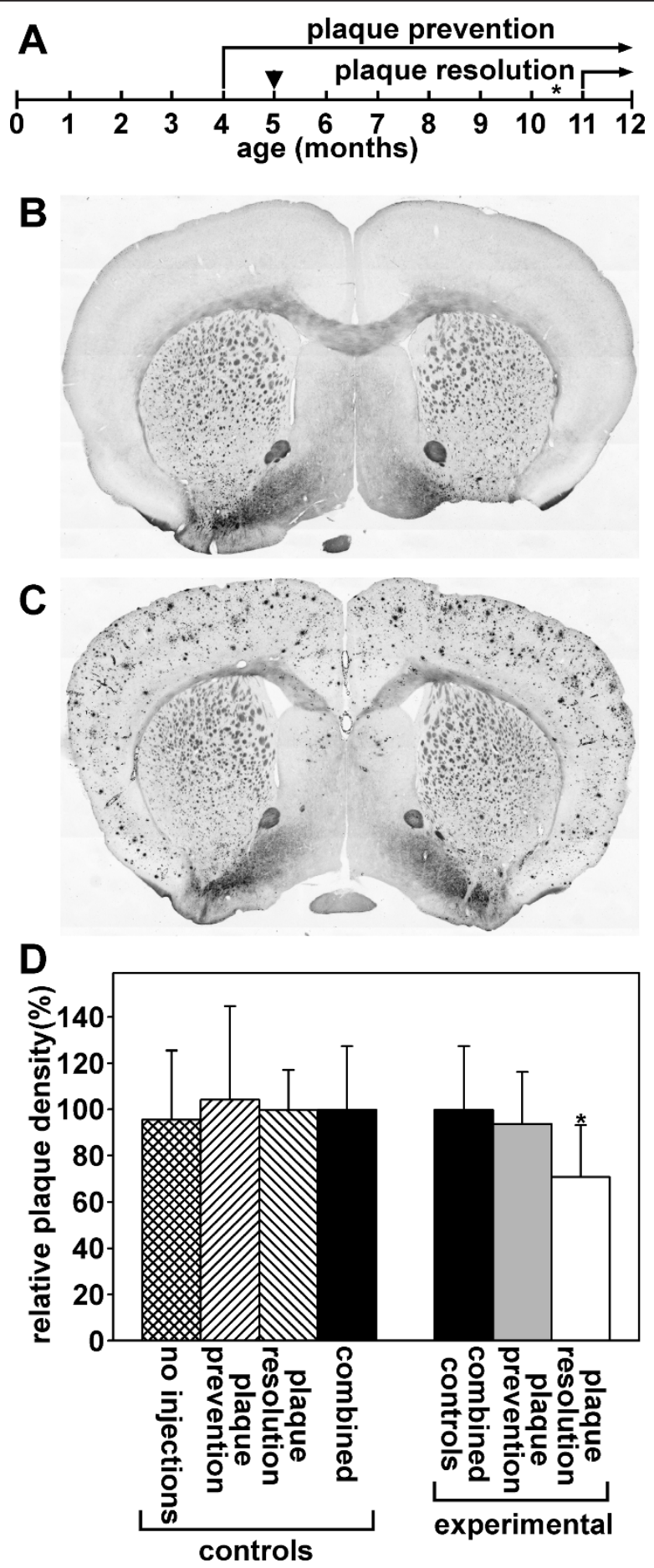

Figure 2 Amyloid plaques in brains of mice and the effect of curcuminoid injections. (A) Time course of curcuminoid injections into Alzheimer transgenic mice. Arrowhead denotes the time point when serum-solubilized curcuminoids were switched to HP- $\gamma$-CD-solubilized curcuminoids, asterisk when i.v. injections were replaced with s.c. injections. (B) Silver stained brain section from a control non-transgenic mouse without plaques. (C) Comparable section from an Alzheimer transgenic mouse at the age of 12 months. (D) The effect of curcuminoid injections on amyloid plaque load. Left panel: Alzheimer transgenic control mice were analyzed at 12 months of age and received either no injections $(n=3)$, or injection of vehicle alone according to the protocols for plaque prevention $(n=3)$ or plaque resolution $(n=3)$. The differences in the relative plaque levels in each of the groups were not statistically different and the values were combined and assigned the value of $100 \%$ $(n=9)$. Right panel: The combined control group was compared to experimental mice that had been injected for plaque prevention $(n=6)$ or plaque resolution $(n=7)$ with a statistical difference defined at the level of $P<0.05\left({ }^{*} P=0.0496\right)$. 
experimental groups (Figure 2D). The plaque load of mice that had received weekly injections of curcuminoids for eight months for plaque prevention, showed no significant difference from the control group, although the overall average was slightly reduced. Mice that received bi-weekly injections of curcuminoids at a four-fold higher concentration for four weeks for plaque resolution, had a statistically significant $(P<0.05)$ lower plaque load at approximately $70 \%$ of the control value (Figure 2D).

These results demonstrate that sporadic injections of curcuminoids did not result in the panacea initially hoped for. To design more effective injection protocols with the goal of achieving a more robust response, the fate of curcuminoids after both i.v. and s.c. injections was further examined.

\section{Curcuminoid levels in plasma and brain after intravenous} and subcutaneous injection

After i.v. injection, curcuminoids and selected metabolites were measured in plasma and brain (Figure 3) from both wild type and transgenic $\mathrm{C} 57 \mathrm{BL} / \mathrm{CH} 3$, as well as $\mathrm{CV}-1$ mice. Since the data from both strains of mice were indistinguishable beyond what would be attributable to individual variations, the results were combined for statistical purposes. Unmodified curcuminoids and their sulfate and glucuronide conjugates were detected in plasma at a $427 \mathrm{~nm}$ absorption wavelength (Figure 3A). The hexa- and octahydrocurcuminoid reduction products were detected in both plasma and brain at the $280 \mathrm{~nm}$ wavelength (Figure 3B). Significant amounts of di- and tetrahydrocurcuminoids were not observed. In addition, conjugates of reduction products or diconjugate curcuminoids could not be reliably determined, either because their concentration was too low or their absorption peaks overlapped with those from numerous co-extracted unrelated compounds that eluted close to the front.

Initial plasma concentration of curcuminoids reached a high level of approximately $100 \mu \mathrm{M}$ immediately following i.v. injection (Figure 4A), which declined rapidly to essentially undetectable levels within 20 minutes. Both conjugation and reduction products were also transiently observed in plasma at concentrations below $20 \mu \mathrm{M}$ (Figure 4A, B). Curcuminoid sulfates and glucuronides in plasma occurred concurrently in individual mice, but their relative levels varied widely between animals and time after injection. Overall, the level of sulfates peaked early and declined to low levels by 15 minutes while glucuronides persisted longer and were detected for up to $1 \mathrm{~h}$. The primary reduction products in plasma were hexahydrocurcuminoids and these persisted for at least 30 minutes. Octahydrocurcuminoids were detected transiently at low levels between 20 and 30 minutes after injection (Figure 4B).
After s.c. injection, peak plasma curcuminoid levels were delayed compared to i.v. injection and they gradually reached a maximum after 30 minutes at $23 \mu \mathrm{M}$. Lower levels of curcuminoids (approximately $1 \mu \mathrm{M}$ ) could be detected for up to $4 \mathrm{~h}$. The peak level of curcuminoid conjugates occurred with a delay compared to the parental compounds at $1 \mathrm{~h}$ after s.c. injection (Figure 5A). The primary monoconjugates were consistently glucuronides, which at peak levels were about five-fold higher than sulfates (Figure 5B). Significant amounts of reduction products were not observed after s.c. injection.

In brain tissue, the highest levels of curcuminoids (approximately $47 \mathrm{nmol} / \mathrm{g}$ ) occurred with a slight delay compared to plasma at two minutes after i.v. injection. No curcuminoid conjugates were observed, which indicated that contamination with blood was insignificant.

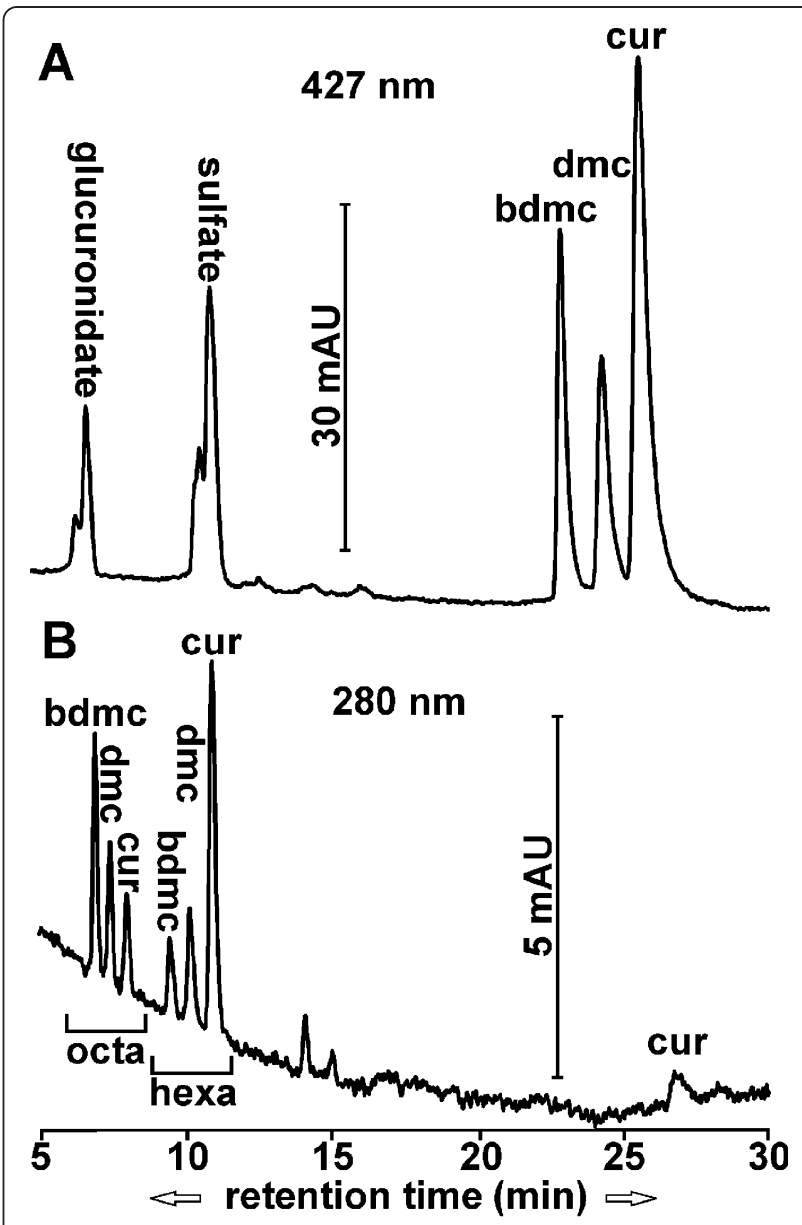

Figure 3 Elution profiles of curcuminoids and metabolites in mice after i.v. injections. (A) Chromatogram (427 nm) of curcuminoids and monoconjugates of sulfate and glucuronide extracted from plasma five minutes after injection. (B) Chromatogram (280 nm) of octahydro- and hexahydrocurcuminoids extracted from brain 15 minutes after injection. 


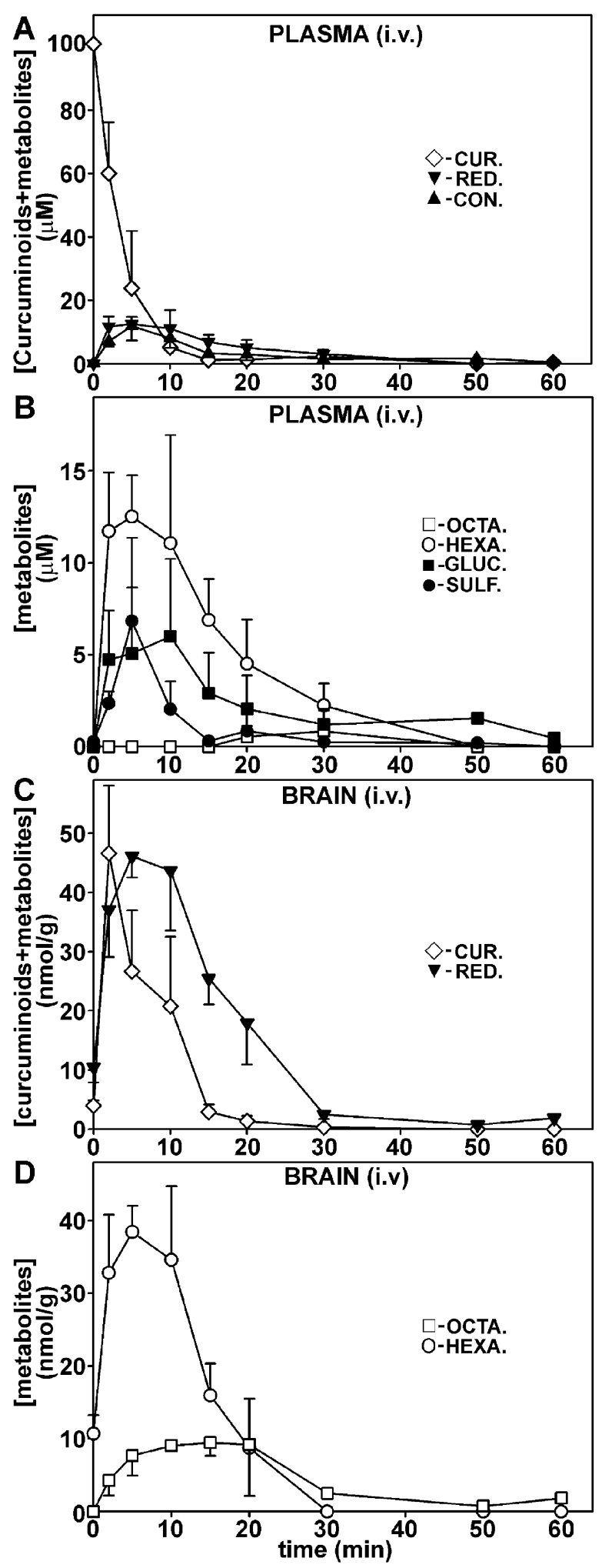

Figure 4 Concentration of curcuminoids and metabolites in plasma (uM) and brain ( $\mathbf{n m o l} / \mathbf{g}$ ) after i.v. injection. (A) Time course of total curcuminoids $(\diamond)$, reduction products (hexa- + octacurcuminoids, $\mathbf{v}$ ), and monoconjugates (sulfates + glucuronides, $\mathbf{\Delta}$ ) in plasma. (B) Time course of individual metabolite concentrations in plasma: hexahydrocurcuminoids (O), octahydrocurcuminoids ( $\square$ ), curcuminoid monoglucuronides $(\mathbf{-})$, and curcuminoid monosulfates $(\bullet)$. (C) Time course of total curcuminoids $(\diamond)$ and reduction products $(\mathbf{v})$ in brain. (D) Time course of hexahydro- (O) and octahydrocurcuminoids (ㅁ) in brain. 


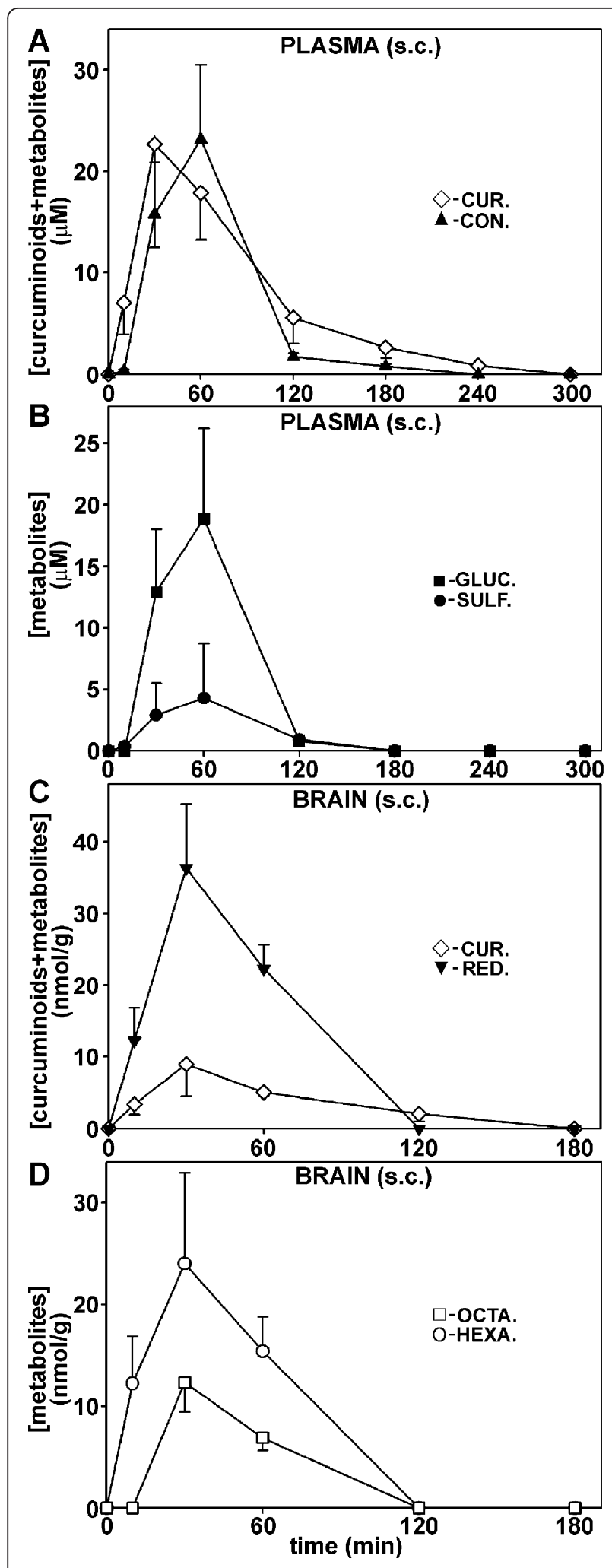

Figure 5 Concentration of curcuminoids and metabolites in plasma $(\mu \mathrm{M})$ and brain $(\mathrm{nmol} / \mathrm{g})$ after s.c. injection. (A-D) Same as depicted in Figure 4, except that significant concentrations of curcuminoid reduction products were not detected in plasma.
While the concentration of curcuminoids declined to undetectable levels after 30 minutes, the reduction products could be detected for up to $1 \mathrm{~h}$ (Figure 4C). Hexahydrocurcuminoids were the main reduction products in the brain, with peak concentrations reaching approximately $40 \mathrm{nmol} / \mathrm{g}$ at five minutes after injection. The levels of octahydrocurcuminoids were lower but persisted for up to $1 \mathrm{~h}$ (Figure 4D).

After s.c. injection, brain curcuminoid levels were considerably lower (approximately $8 \mathrm{nmol} / \mathrm{g}$ ) than after i.v. injection (Figure $5 \mathrm{C}$ ) with a maximum attained at 30 minutes. However, levels above $1 \mathrm{nmol} / \mathrm{g}$ were observed for more than $2 \mathrm{~h}$, whereas similar concentrations were already reached within 20 minutes after i.v. injection. In contrast, the levels of reduction products (approximately $36 \mathrm{nmol} / \mathrm{g}$ ) were comparable to those observed after i.v. injection (approximately $46 \mathrm{nmol} / \mathrm{g}$ ). Similar to i.v. injection, octahydrocurcuminoids appeared with a slight delay compared to hexahydrocurcuminoids. Nevertheless, all reduction products were essentially undetectable after $2 \mathrm{~h}$ following s.c. injection (Figure 5D).

This demonstrates that significant levels of curcuminoids can initially be achieved in plasma and brain after both i.v. and s.c. injection. However, these levels are transient and although the curcuminoid levels decline somewhat more slowly after s.c injection, ultimately they persist at detectable concentrations for no longer than two to four hours. These results further suggest extensive binding of injected curcuminoids to cells and tissues, followed by rapid local metabolic conversion and gradual release into the circulation. In addition, the time course of the appearance of metabolites in the brain indicates sequential reduction from hexahydrocurcuminoids to octahydrocurcuminoids and this aspect was examined in further detail.

\section{Sequential generation of curcuminoid reduction products} in brain after i.v. injection

It takes at least several minutes before brains can be removed and cooled to the point that further metabolism may be considered negligible. To investigate how postmortem processes contribute to the metabolism of curcuminoids, a mouse was subjected to cardiac perfusion with HP- $\gamma$-CD-solubilized curcuminoids diluted with serum to a similar concentration as that achieved by i.v injections (5\%). The total amount of curcuminoids extracted from a brain after perfusion was approximately $256 \mathrm{nmol} / \mathrm{g}$ and this may be considered the maximum capacity for uptake/binding within these concentration parameters. Comparable values in other organs were $1,383 \mathrm{nmol} / \mathrm{g}$ for the liver, $579 \mathrm{nmol} / \mathrm{g}$ for the kidney and $287 \mathrm{nmol} / \mathrm{g}$ for the small intestine. The primary reduction products observed in brains after perfusion were hexahydrocurcuminoids at a concentration 
of approximately $16 \mathrm{nmol} / \mathrm{g}$ and no quantifiable amounts of octahydrocurcuminoids (Figure 6A). A similar pattern was observed in the other organs, except that a small additional amount of glucuronidation was

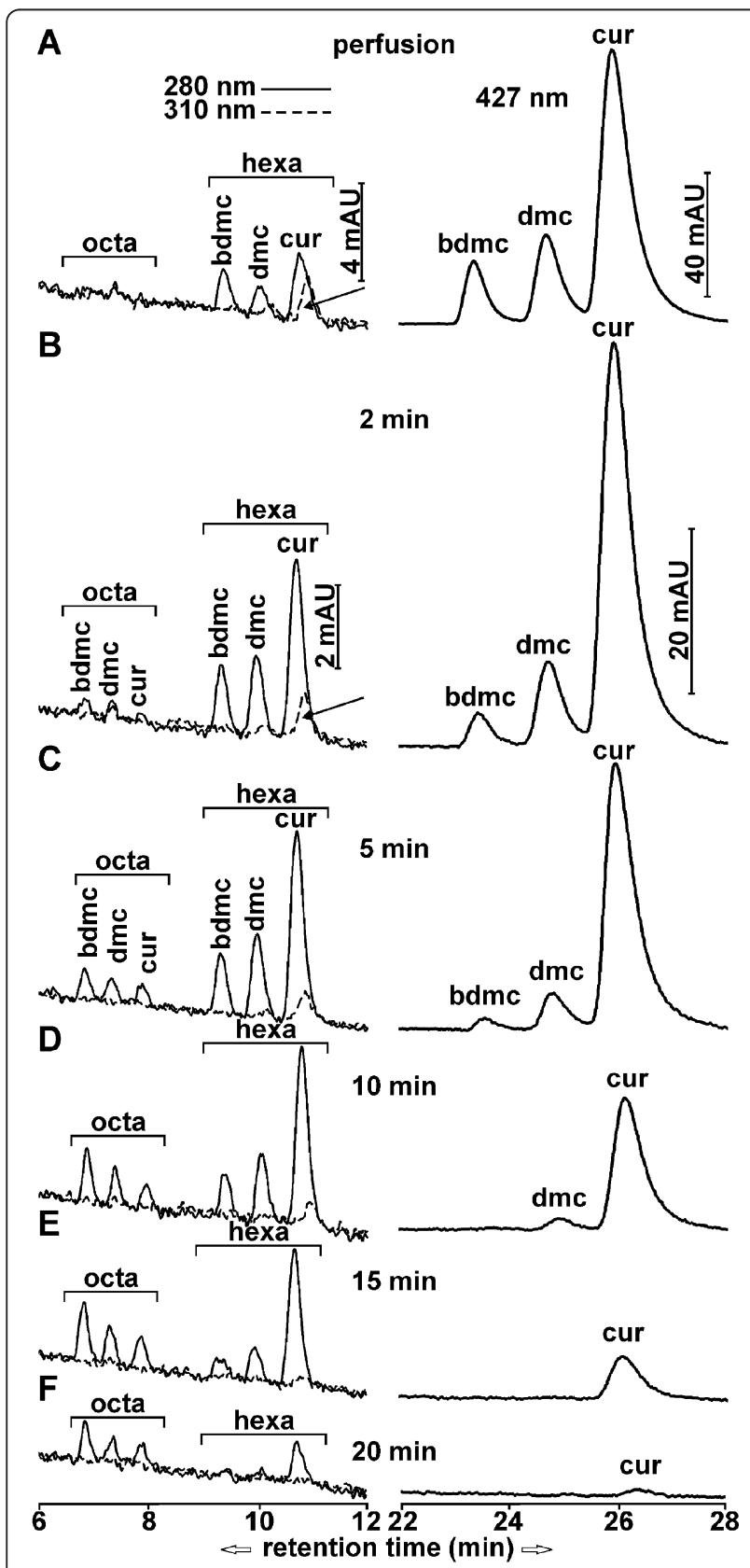

Figure 6 Chromatograms of curcuminoids and reduction products in brain after cardiac perfusion and i.v. injection. Curcuminoids (right panels, retention time 22 to 28 minutes) were monitored at wavelength $427 \mathrm{~nm}$ and reduction products (left panels, retention time 6 to 12 minutes) at wavelengths $280 \mathrm{~nm}$ (solid lines) and $310 \mathrm{~nm}$ (dashed lines). The position of hexa- and

octahydrocurcuminoids are indicated by brackets. (A) Elution profiles after cardiac perfusion. (B-F) Elution profiles at 2 minutes (B), 5 minutes (C), 10 minutes (D), 15 minutes (E) and 20 minutes (F) after i.v. injection. detected in the liver (not shown). This suggests that postmortem reduction is blocked at the hexahydrocurcuminoid stage. However, in addition to the hexahydrocurcuminoid peaks at $280 \mathrm{~nm}$ wavelength, further peaks were observed at $310 \mathrm{~nm}$ wavelength. The tentative identity of these compounds was previously established in an embryonal carcinoma cell line [36] and they are here designated as dihydrocurcuminoidols, which may represent alternative intermediates in the curcuminoid reduction pathway (Figure 7)

Under in vivo conditions, the brain content of curcuminoids declined to essentially undetectable levels within 20 minutes after i.v. injection, concomitant with a sequential accumulation of reduction products. Hexahydrocurcuminoids appeared rapidly within 2 minutes and reached a maximum after 5 to 10 minutes. In contrast, octahydrocurcuminoids emerged later with peak concentrations after 10 to 15 minutes. The relative levels of hexahydrocurcuminoids reflected those of the parental curcuminoids in the succession CUR > DMC > BDMC, whereas the relative levels of octahydrocurcuminoids were reversed. Dihydrocurcuminoidols, were also detected here, albeit at lower relative levels (Figure 6B-F).

\section{Differential cellular curcuminoid binding upon solubilization in serum or HP- $\gamma-\mathrm{CD}$}

Cellular curcuminoid binding as it is affected by the solubilization vehicles and their dilution with mouse serum was evaluated in cultured NT2/D1 cells, in which these parameters have been extensively characterized [36]. Binding dose curves were generated by incubating NT2/D1 cells with increasing amounts of curcuminoids solubilized

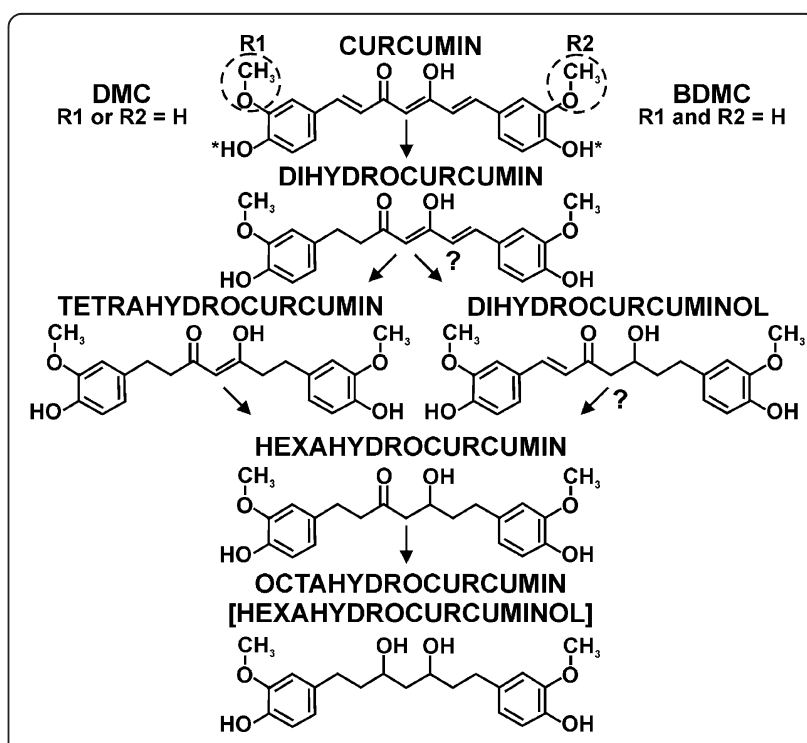

Figure 7 Structure of curcuminoids and putative alternative reduction pathways. 
in either mouse serum or $10 \% \mathrm{HP}-\gamma-\mathrm{CD}$. The binding dose curves yielded an apparent $K_{D}$ of $14.88 \mu \mathrm{M}$ for mouse serum- and $7.58 \mu \mathrm{M}$ for HP- $\gamma$-CD-solubilized curcuminoids (Figure 8A). A similar dose curve established with curcuminoids solubilized in FCS, resulted in an apparent $K_{D}$ of $9.16 \mu \mathrm{M}$ (not shown). Nonspecific binding was more pronounced with serum-solubilized than with HP- $\gamma$ $\mathrm{CD}$-solubilized curcuminoids. Since it can be assumed that the cellular binding affinity for curcuminoids per se remains constant, the variations in the apparent binding

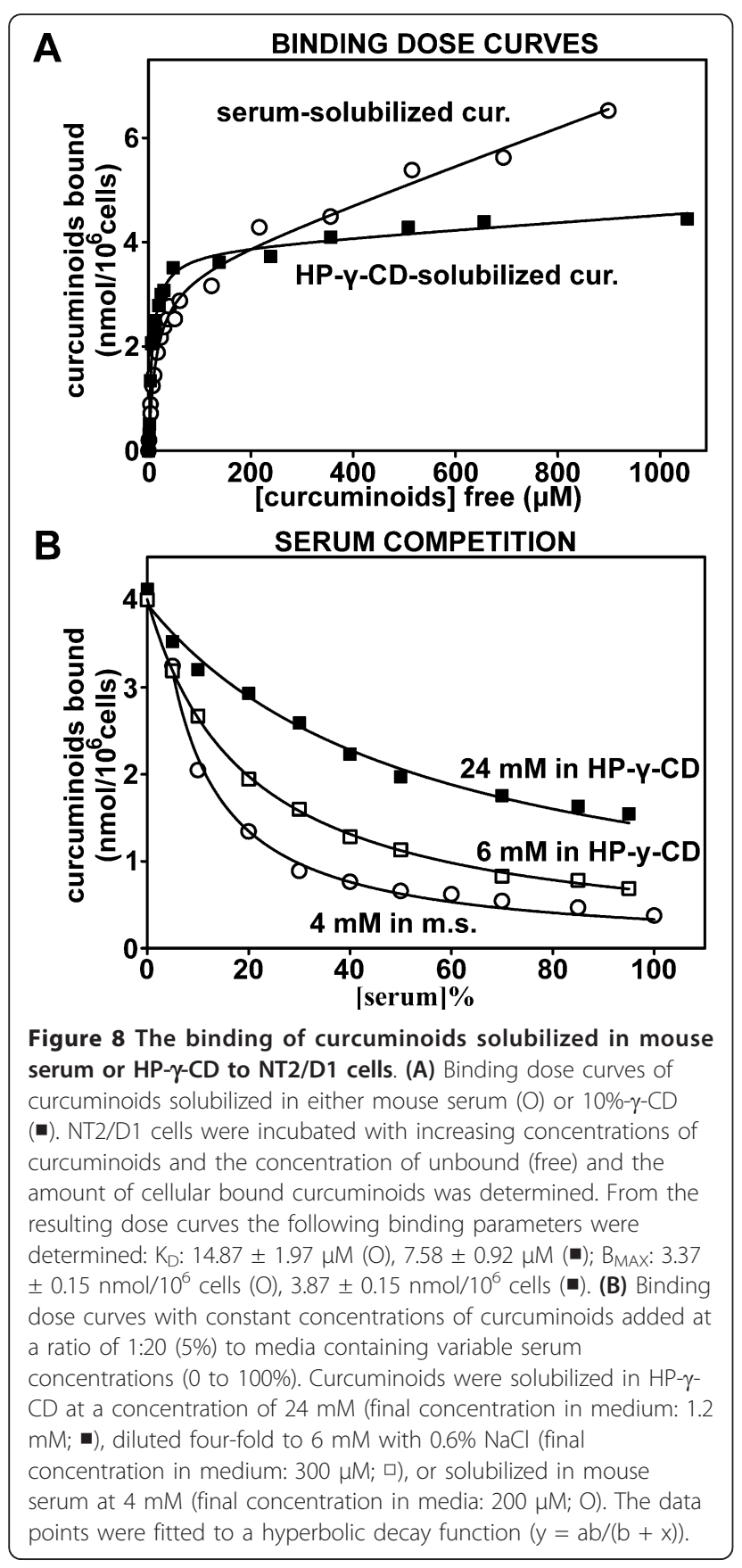

$\mathrm{K}_{\mathrm{D}} \mathrm{s}$ may be interpreted as differences in affinities between curcuminoids and solubilization vehicles. However, the binding dose curves were generated with increasing amounts of pure solubilized curcuminoids and this does not reflect the situation in vivo, where the i.v. injected curcuminoids are immediately diluted with excess blood. The influence of serum dilution on cellular binding was, therefore, further examined to reflect this circumstance.

Curcuminoid preparations originally used for i.v. injections $(24 \mathrm{mM}$ and $6 \mathrm{mM} \mathrm{HP}-\gamma$-CD-solubilized, $4 \mathrm{mM}$ mouse serum-solubilized) were diluted 1:20 with DMEM or substituted with increasing proportions of mouse serum without curcuminoids. This resulted in total serum concentrations ranging from 0 to $95 \%$ for curcuminoids solubilized in HP- $\gamma-\mathrm{CD}$ and 5 to $100 \%$ for serum-solubilized curcuminoids. In all cases, cellular binding decreased with increasing serum concentrations in a manner consistent with a hyperbolic decline as described elsewhere [36] (Figure 8B). However, the decline in binding with increasing serum concentrations was dependent on the concentration of the curcuminoids in the media. Therefore, at maximal serum concentrations cellular binding remained at 39\% (24 $\mathrm{mM}$ curcuminoids), $15 \%(6 \mathrm{mM})$ and $12 \%(4 \mathrm{mM})$ relative to the respective binding without added serum. These results are consistent with the notion that serum components, foremost albumin [29], act as competitors for cellular binding via the affinities of their interaction domains, which retain the curcuminoids in solution.

Differences in cellular binding of individual curcuminoids were also observed, depending on whether they were solubilized in serum (Figure 9A) or HP- $\gamma$-CD (Figure 9B). At lower curcuminoid concentrations within the vicinity of the $K_{D}$ values $\left(50 \% B_{\text {MAX }}\right.$ Figure $9 A$, B, left panels), the distribution of cellular curcuminoids largely reflected the composition of the curcuminoids in the respective media (compare Figure 1A). However, under saturating conditions ( $\geq 100 \% \mathrm{~B}_{\mathrm{MAX}}$; Figure $9 \mathrm{~A}, \mathrm{~B}$, middle panels), which represents the curcuminoid concentrations employed in the serum competition experiments (Figure $8 \mathrm{~B}$ ), the primary component bound to cells incubated with curcuminoids solubilized in serum was CUR (61\%), whereas BDMC (48\%) predominated in cells incubated with curcuminoids solubilized in HP- $\gamma$ CD. Indeed, both CUR and BDMC were the main contributors to nonspecific cellular binding during incubation with the respective solubilization vehicles. During serum competition of curcuminoids solubilized in serum, cellular binding decreased concordantly so that the relative distribution of bound curcuminoids remained unchanged (Figure 9A, right panel). In contrast, serum competition of curcuminoids solubilized in HP- $\gamma$-CD reduced cellular binding of BDMC more robustly so that the relative 


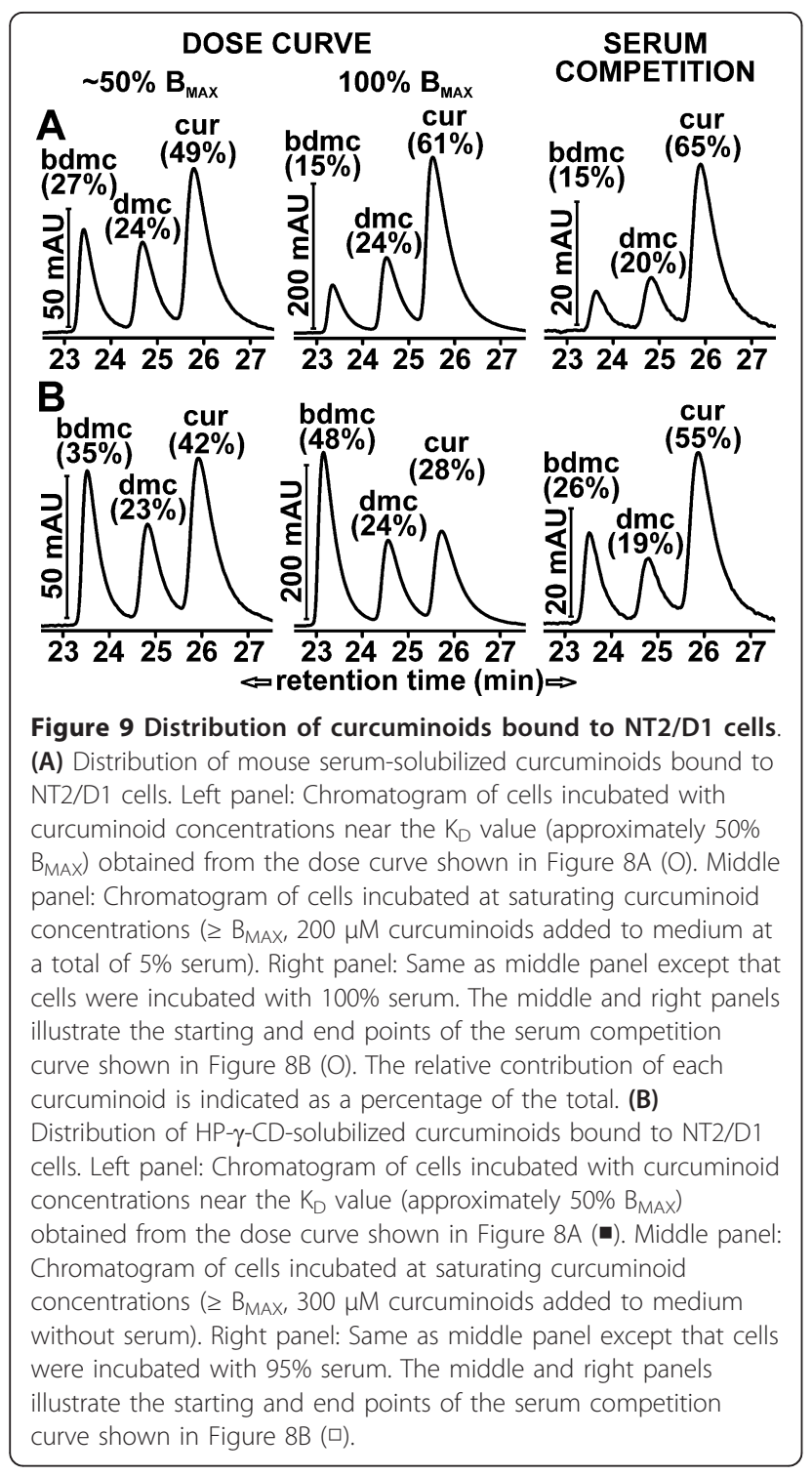

distribution of bound curcuminoids was shifted toward CUR (Figure 9B, right panel).

Overall, these results indicate that the relative affinity of curcuminoids for HP- $\gamma-\mathrm{CD}$ is weaker than for mouse serum components, which causes a reduction in the apparent cellular binding $K_{D}$. Furthermore, the relative affinity of BDMC for HP- $\gamma-\mathrm{CD}$ is weaker than that of CUR, which causes this component to preferentially bind to cells at higher curcuminoid concentrations. The preferential serum competition of cellular binding of BDMC in media containing curcuminoids solubilized in HP- $\gamma-C D$ is consistent with this idea. In general, the differences in cellular binding characteristics observed between curcuminoids solubilized in HP- $\gamma-\mathrm{CD}$ or serum are primarily noticed when these are used in their pure form. Upon dilution with serum and at lower curcuminoid concentrations, which are conditions rapidly established in vivo, these differences become minimized.

\section{Discussion}

The oral administration of curcumin to humans, mice and rats has resulted in plasma levels typically not exceeding $1 \mu \mathrm{M}$ concentrations and similarly low tissue levels (for recent reviews and references therein see: $[26-28,44])$. Alternatively, curcumin has been delivered to mice or rats by intraperitoneal (i.p) injection at dosages of 6 to $100 \mathrm{mg} / \mathrm{kg}$ body weight [15,45-47]. For this purpose, curcumin was either dissolved in DMSO [45-47] or in $\mathrm{NaOH}$ followed by neutralization [15]. In those studies, peak curcumin plasma concentrations were within the range of approximately 3.5 to $25 \mu \mathrm{M}$ and brain levels approximately 1 to $2 \mathrm{nmol} / \mathrm{g}$. After i.v. injection in rats of $10 \mathrm{mg} / \mathrm{kg}$ curcumin solubilized in a cocktail containing DMA/PEG/dextrose, initial plasma concentrations of approximately $27 \mu \mathrm{M}$ were reported [46]. Similar results were reported in another study in which curcumin had been solubilized in glycerol formal and i.v. injected at a dosage of $40 \mathrm{mg} / \mathrm{kg}$ [48]. By comparison, in the present study mice were i.v. injected with $0.1 \mathrm{ml}$ of $24 \mathrm{mM}$ curcuminoids solubilized in 10\% HP- $\gamma$-CD. This represents a total dose of $0.84 \mathrm{mg}$ or approximately $33 \mathrm{mg} / \mathrm{kg}$ curcuminoids. Under these conditions, initial curcuminoid plasma concentrations of about $100 \mu \mathrm{M}$ were attainable. Adjusted for the total dose applied, these concentrations are similar to those observed for the rat [46], but they resulted in transient brain concentrations of approximately $47 \mathrm{nmol} / \mathrm{g}$ (Figure 4), which were higher than those reported in any other study. Similarly, a four-fold higher curcuminoid dose (approximately $134 \mathrm{mg} / \mathrm{kg}$ ) administered by s.c. injection yielded maximal plasma concentrations of approximately $23 \mu \mathrm{M}$ and brain levels of approximately $8 \mathrm{nmol} / \mathrm{g}$. Although these amounts are lower than those achieved by i.v. injection, the parental curcuminoids were released gradually from the injection site and they persisted longer in both plasma and brain (Figure 5). These levels are also higher than those typically observed after i.p. injection (see above). However, in one study curcumin was administered at a much lower dose $(3 \mathrm{mg} / \mathrm{kg})$ and this yielded relatively high brain levels of approximately $3.2 \mathrm{nmol} / \mathrm{g}$ at four hours after intramuscular injection [15].

The injection protocol presented here combines the high solubility of curcuminoids in 10\% HP- $\gamma$-CD with the relatively low level of toxicity of the carrier vehicle. In addition, HP- $\gamma$-CD does not cause apparent immune reactions that are associated with the use of heterologous serum to solubilize curcuminoids. However, at the highest doses used for i.v. injection $(33 \mathrm{mg} / \mathrm{kg})$, toxic reactions do develop and these may represent the 
maximum amount of curcuminoids tolerated before lethal effects occur. After a single i.v. injection, high plasma levels are exceedingly transient and the concentration of native curcuminoids drops to insignificant levels within 20 minutes (Figure 4). The decline in the level of circulating curcuminoids can be considered due to rapid metabolism combined with widespread binding/ uptake to cells and tissues.

The binding of curcuminoids to cells in culture has been described in detail elsewhere [36]. Those studies were expanded to include preparations employed here, where curcuminoids had been sequentially solubilized in mouse serum and 10\% HP- $\gamma$-CD (Figure 8). This was done by first adding curcuminoids as a solid powder followed by the addition of DMSO-dissolved curcuminoids. This method results in a maximal solubility of curcuminoids and a relatively balanced composition [29]. The apparent binding $\mathrm{K}_{\mathrm{D}} \mathrm{S}$ in these preparations were higher for curcuminoids solubilized in mouse serum (14.88 $\mu \mathrm{M})$ than in FCS $(9.16 \mu \mathrm{M})$. Since mouse serum has the capacity to solubilize higher concentrations of curcuminoids than FCS ( 3 to $4 \mathrm{mM}$ vs. approximately $1.7 \mathrm{mM}$ [29]), it is likely that these differences in binding $K_{D} S$ reflect differences in affinities or concentrations between the curcuminoid interaction domains in serum components, as it may be reasonably assumed that the cellular binding affinities remain constant. It is further expected that curcuminoids solubilized in sera from different species or possibly different preparations from the same species, will produce different apparent cellular binding $\mathrm{K}_{\mathrm{D}} \mathrm{S}$ due to their variable serum compositions. In addition, alternative solubilization vehicles may have differential affinities for individual curcuminoids. This is exemplified by the cellular binding of individual curcuminoids solubilized in HP- $\gamma$-CD (Figure 9). Although curcuminoids solubilized in either HP- $\gamma$-CD or serum have similar compositions (Figure 1), the primary curcuminoids bound to cells at saturating concentrations were BDMC and CUR, respectively. However, upon dilution with excess serum, both preparations converged to the same compositions of cellular-bound curcuminoids (Figure 9). Although these observations were made with cultured cells, it is likely that similar binding occurs in vivo. Indeed, the extensive binding of curcuminoids to brain (Figure 6) and other organs (not shown) shows a similar distribution pattern as that obtained with cultured cells incubated with curcuminoids and excess serum. Except for considerations relating to solubilization capacity, toxicity or immune reactions, it is in this case irrelevant whether curcuminoids are solubilized in serum or HP- $\gamma$-CD.

The metabolic conversion of curcuminoids after i.v. injection is rapid and it includes both conjugation and reduction products (Figure 4). Since conjugation takes place in the liver, intestines and kidneys, the resulting products are observed primarily in plasma but also at the sites of excretion [48-51]. In addition to conjugation products, the hexa- and octahydrocurcuminoid reduction products are also prominently represented in the circulation. It is likely that these are primarily contributed by curcuminoids that were taken up by peripheral cells and tissues, metabolized locally, and subsequently released into the bloodstream. In contrast, after s.c. injection the curcuminoid reduction products are not readily detected in plasma. This is most likely due to the more gradual release from tissues followed by rapid excretion. Although only the monoconjugates of sulfate and glucuronide were investigated here, it is likely that mixed diconjugates are also produced $[48,50,51]$. Some studies have reported the formation of tetrahydrocurcumin in mice $[47,49]$, which was not detected in this study. Instead, the hexa- and octahydrocurcuminoids predominated. In addition, different reduction products with different physical characteristics, here referred to as dihydrocurcuminoidols, were detected in the brain. These compounds were first identified as the final reduction products in the teratocarcinoma cell line NT2/D1 [36], while its presence in vivo has not been previously reported. However, dihydrocurcuminoidols are most prominently produced after perfusion under post-mortem conditions. Their timedependent production in the brain following i.v. injection also seems to be correlated with the amount of unmodified curcuminoids present. It can, therefore, not be excluded that this is a post-mortem effect that rapidly occurs during the removal of tissues. The enzyme systems responsible for the reductive conversion of curcuminoids have not yet been conclusively identified. However, it appears that octahydrocurcuminoids are sequentially generated from hexahydrocurcuminoids. For example, during perfusion only hexahydrocurcuminoids together with smaller amounts of dihydrocurcuminoidols were generated and since the post-mortem conversion to octahydrocurcuminoids was blocked, there was a larger relative accumulation of hexahydro-BDMC compared to in vivo conditions (Figure 6). This indicates that the overall conversion to octahydrocurcuminoids requires distinct enzyme systems with different substrate specificities and metabolic requirements. The more efficient generation of octahydro-BDMC than octahydro-CUR is consistent with this notion, as is the gender-specific generation of reduction products in the rat [39]. The curcumin reducing enzymes have also been found to be distributed between cytoplasmic and microsomal compartments [38]. In addition, different cell lines in culture produce different reduction products. For example, in the astrocytoma cell line CCF-STTG1, reduction proceeds to the octahydrocurcuminoid stage, whereas in HeLa cells hexahydrocurcuminoids are the end products [36]. These cell 
lines also produce varying amounts of dihydrocurcuminoidols [36]. Based on these observations, possible alternative reduction pathways for curcuminoid reduction are proposed (Figure 7).

\section{Conclusions}

This study was undertaken to examine the feasibility of using an injectable form of curcuminoids to modulate the formation of amyloid plaques in brains of Alzheimer transgenic mice. This was based on the premise that relatively low concentrations of curcumin suffice to eliminate or reverse amyloid fibril formation in vitro. Furthermore, a number of studies have reported a reduction in plaque load following long-term (four to nine months) oral uptake of curcumin in similar transgenic mouse models. Despite low plasma levels, the plaque load was reduced to 48 to $69 \%$ of control values [9,15-17]. However, including curcumin in the diet represents chronic exposure to low levels of curcumin. In this study, the amount of curcuminoids injected was relatively high, yielding a significant rapid distribution into both plasma and tissues. Nevertheless, the high rate of metabolism rendered these levels short-lived. In effect, the long-term intermittent exposure at intervals of once/week had no discernable influence on plaque formation, although at four-fold higher dosage and with a frequency of twice/week for one month, a reduction in plaque load to about $70 \%$ of control values was observed. Indeed, similar plaque load reductions were reported in the same strain of mice after daily tail vein injections for seven days using only $7.5 \mathrm{mg} / \mathrm{kg}$ curcumin [8]. It is, therefore, conceivable that inhibition of plaque formation and plaque resolution depends on a more frequent administration of curcuminoids. Since frequent long-term tail vein injections in mice are problematic, these could be augmented or substituted with better tolerated subcutaneous injections in future studies. These modes of administration could also be complemented with formulations for improved oral uptake. For example, preliminary studies with HP- $\gamma$-CD-solubilized curcuminoids in drinking water yielded consistent plasma concentrations in the range of 0.5 to $1 \mu \mathrm{M}$ (not shown). Therefore, combining the oral uptake of curcuminoids via both food and drinking water with injection protocols may result in more effective procedures for plaque prevention and elimination.

\section{Abbreviations}

AD: Alzheimer's disease; APP: amyloid protein precursor; BDMC: bisdemethoxycurcumin; CUR: curcumin; DMA, N: N-dimethylacetamide; DMC: demethoxycurcumin; DMEM: Dulbecco's Minimal Essential Medium; DMSO: dimethyl sulfoxide; FCS: fetal calf serum; HP- $y$-CD: 2-hydroxypropyl- $\gamma$ cyclodextrin; i.v.: intravenous; PBS: phosphate-buffered saline; PEG: polyethylene glycol; s.c.: subcutaneous

\section{Authors' contributions}

All authors contributed to the manuscript. WQ devised the protocols and performed the biochemical experiments. NS and JR carried out all animal related procedures. All authors read and approved the final manuscript.

\section{Competing interests}

The authors declare that they have no competing interests.

\section{Acknowledgements}

This research was supported by grant A2009342 from the American Health Assistance Foundation. Mass spectrometry was performed by Robert Rieger at the Proteomics Center of the State University of New York at Stony Brook, which was supported by the shared instrumentation grant NIH/NCRR 1 S10 RR023680-1. Brains were processed for silver-staining at the facilities of NeuroScience Associates (Knoxville, TN, USA).

\section{Author details}

${ }^{1}$ Department of Psychiatry and Behavioral Science, Stony Brook University Medical Center, 100 Nicolls Road, Stony Brook, New York 11794-8101, USA. ${ }^{2}$ Division of Laboratory Animal Resources, Stony Brook University Medical Center, 100 Nicolls Road, Stony Brook, New York 11794-8611, USA.

Received: 8 January 2013 Revised: 9 March 2013

Accepted: 18 March 2013 Published: 28 March 2013

\section{References}

1. Braga ME, Leal PF, Carvalho JE, Meireles MA: Comparison of yield, composition, and antioxidant activity of turmeric (Curcuma longa L.) extracts obtained using various techniques. J Agric Food Chem 2003, 51:6604-6611.

2. Tayyem RF, Heath DD, Al-Delaimy WK, Rock CL: Curcumin content of turmeric and curry powders. Nutr Cancer 2006, 55:126-131.

3. Jayaprakasha GK, Jagan Mohan Rao L, Sakariah KK: Improved HPLC method for the determination of curcumin, demethoxycurcumin, and bisdemethoxycurcumin. J Agric Food Chem 2002, 50:3668-3672

4. Hiserodt R, Hartman TG, Ho CT, Rosen RT: Characterization of powdered turmeric by liquid chromatography-mass spectrometry and gas chromatography-mass spectrometry. J Chromatogr A 1996, 740:51-63.

5. Yanagisawa D, Shirai N, Amatsubo T, Taguchi H, Hirao K, Urushitani M, Morikawa S, Inubushi T, Kato M, Kato F, Morino K, Kimura H, Nakano I, Yoshida C, Okada T, Sano M, Wada Y, Wada KN, Yamamoto A, Tooyama I: Relationship between the tautomeric structures of curcumin derivatives and their A $\beta$-binding activities in the context of therapies for Alzheimer's disease. Biomaterials 2010, 31:4179-4185.

6. Mutsuga M, Chambers JK, Uchida K, Tei M, Makibuchi T, Mizorogi T, Takashima A, Nakayama $\mathrm{H}$ : Binding of curcumin to senile plaques and cerebral amyloid angiopathy in the aged brain of various animals and to neurofibrillary tangles in Alzheimer's brain. J Vet Med Sci 2012, 74:51-57.

7. Mohorko N, Repovs G, Popovic M, Kovacs GG, Bresjanac M: Curcumin labeling of neuronal fibrillar tau inclusions in human brain samples. J Neuropathol Exp Neurol 2010, 69:405-414.

8. Garcia-Alloza M, Borrelli LA, Rozkalne A, Hyman BT, Bacskai BJ: Curcumin labels amyloid pathology in vivo, disrupts existing plaques, and partially restores distorted neurites in an Alzheimer mouse model. J Neurochem 2007, 102:1095-1104.

9. Yang F, Lim GP, Begum AN, Ubeda OJ, Simmons MR, Ambegaokar SS, Chen PP, Kayed R, Glabe CG, Frautschy SA, Cole GM: Curcumin inhibits formation of amyloid $\beta$ oligomers and fibrils, binds plaques, and reduces amyloid in vivo. J Biol Chem 2005, 280:5892-5901.

10. Liu K, Guo TL, Chojnacki J, Lee HG, Wang X, Siedlak SL, Rao W, Zhu X, Zhang S: Bivalent ligand containing curcumin and cholesterol as fluorescence probe for $A \beta$ plaques in Alzheimer's disease. ACS Chem Neurosci 2012, 3:141-146.

11. Ono K, Hasegawa K, Naiki H, Yamada M: Curcumin has potent antiamyloidogenic effects for Alzheimer's $\beta$-amyloid fibrils in vitro. J Neurosci Res 2004, 75:742-750.

12. Kim H, Park BS, Lee KG, Choi CY, Jang SS, Kim YH, Lee SE: Effects of naturally occurring compounds on fibril formation and oxidative stress of $\beta$-amyloid. J Agric Food Chem 2005, 53:8537-8541. 
13. Reinke AA, Gestwicki JE: Structure-activity relationships of amyloid $\beta$ aggregation inhibitors based on curcumin: influence of linker length and flexibility. Chem Biol Drug Des 2007, 70:206-215.

14. Chen SY, Chen Y, Li YP, Chen SH, Tan JH, Ou TM, Gu LQ, Huang ZS: Design, synthesis, and biological evaluation of curcumin analogues as multifunctional agents for the treatment of Alzheimer's disease. Bioorg Med Chem 2011, 19:5596-5604.

15. Begum AN, Jones MR, Lim GP, Morihara T, Kim P, Heath DD, Rock CL, Pruitt MA, Yang F, Hudspeth B, Hu S, Faull KF, Teter B, Cole GM, Frautschy SA: Curcumin structure-function, bioavailability, and efficacy in models of neuroinflammation and Alzheimer's disease. J Pharmacol Exp Ther 2008, 326:196-208.

16. Lim GP, Chu T, Yang F, Beech W, Frautschy SA, Cole GM: The curry spice curcumin reduces oxidative damage and amyloid pathology in an Alzheimer transgenic mouse. J Neurosci 2001, 21:8370-8377.

17. Wang YJ, Thomas P, Zhong JH, Bi FF, Kosaraju S, Pollard A, Fenech M, Zhou XF: Consumption of grape seed extract prevents amyloid- $\beta$ deposition and attenuates inflammation in brain of an Alzheimer's disease mouse. Neurotox Res 2009, 15:3-14.

18. Zhang L, Fiala M, Cashman J, Sayre J, Espinosa A, Mahanian M, Zaghi J, Badmaev V, Graves MC, Bernard G, Rosenthal M: Curcuminoids enhance amyloid- $\beta$ uptake by macrophages of Alzheimer's disease patients. J Alzheimers Dis 2006, 10:1-7.

19. Zhang C, Browne A, Child D, Tanzi RE: Curcumin decreases amyloid- $\beta$ peptide levels by attenuating the maturation of amyloid- $\beta$ precursor protein. J Biol Chem 2010, 285:28472-28480.

20. Liu H, Li Z, Qiu D, Gu Q, Lei Q, Mao L: The inhibitory effects of different curcuminoids on $\beta$-amyloid protein, $\beta$-amyloid precursor protein and $\beta$ site amyloid precursor protein cleaving enzyme 1 in swAPP HEK293 cells. Neurosci Lett 2010, 485:83-88.

21. Xiong Z, Hongmei Z, Lu S, Yu L: Curcumin mediates presenilin-1 activity to reduce $\beta$-amyloid production in a model of Alzheimer's disease. Pharmacol Rep 2011, 63:1101-1108.

22. Ma QL, Yang F, Rosario ER, Ubeda OJ, Beech W, Gant DJ, Chen PP, Hudspeth B, Chen C, Zhao Y, Vinters HV, Frautschy SA, Cole GM: $\beta$-amyloid oligomers induce phosphorylation of tau and inactivation of insulin receptor substrate via c-Jun $\mathrm{N}$-terminal kinase signaling: suppression by omega-3 fatty acids and curcumin. J Neurosci 2009, 29:9078-9089.

23. Park SY, Kim HS, Cho EK, Kwon BY, Phark S, Hwang KW, Sul D: Curcumin protected PC12 cells against $\beta$-amyloid-induced toxicity through the inhibition of oxidative damage and tau hyperphosphorylation. Food Chem Toxicol 2008, 46:2881-2887.

24. Wang J, Zhang YJ, Du S: The protective effect of curcumin on $A \beta$ induced aberrant cell cycle reentry on primary cultured rat cortical neurons. Eur Rev Med Pharmacol Sci 2012, 16:445-454.

25. Ahmed T, Gilani AH: A comparative study of curcuminoids to measure their effect on inflammatory and apoptotic gene expression in an $A \beta$ plus ibotenic acid-infused rat model of Alzheimer's disease. Brain Res 2011, 1400:1-18

26. Shen L, Ji HF: The pharmacology of curcumin: is it the degradation products? Trends Mol Med 2012, 18:138-144.

27. Quitschke WW: Bioavailability and metabolism of curcuminoids. In Natural Compounds as Inducers of Cell Death. Volume 1. Edited by: Diedrich M, Noworyta K. Heidelberg: Springer Verlag; 2012:95-125.

28. Hamaguchi T, Ono K, Yamada M: Curcumin and Alzheimer's disease. CNS Neurosci Ther 2010, 16:285-297.

29. Quitschke WW: Differential solubility of curcuminoids in serum and albumin solutions: implications for analytical and therapeutic applications. BMC Biotechnol 2008, 8:84

30. Campell SK, Switzer RC, Martin TL: Alzheimer's plaques and tangles: A controlled and enhanced silver staining method. An abstract submitted for the $17^{\text {th }}$ Annual Meeting of the Society for Neuroscience. SoC Neurosci Abstr 1987, 13:189.9.

31. Switzer RC, Campell SK, Murdock TM: A histolologic method for staining Alzheimer pathology. United States Patent \#5192688 1993.

32. Braak H, Thal DR, Ghebremedhin E, Del Tredici K: Stages of the pathologic process in Alzheimer disease: age categories from 1 to 100 years. $J$ Neuropathol Exp Neurol 2011, 70:960-969.

33. Schönheit B, Zarski R, Ohm TG: Spatial and temporal relationships between plaques and tangles in Alzheimer-pathology. Neurobiol Aging 2004, 25:697-711.
34. Czasch S, Paul S, Baumgärtner W: A comparison of immunohistochemical and silver staining methods for the detection of diffuse plaques in the aged canine brain. Neurobiol Aging 2006, 27:293-305.

35. Braak H, Braak E, Ohm T, Bohl J: Alzheimer's disease: mismatch between amyloid plaques and neuritic plaques. Neurosci Lett 1989, 103:24-28.

36. Quitschke WW: Curcuminoid binding to embryonal carcinoma cells: reductive metabolism, induction of apoptosis, senescence, and inhibition of cell proliferation. PLoS One 2012, 7:e39568.

37. Péret-Almeida L, Cherubino AP, Alves RJ, Dufossé L, Glória MB: Separation and determination of the physico-chemical characteristics of curcumin, demethoxycurcumin and bisdemethoxycurcumin. Food Res Int 2005, 38:1039-1044.

38. Ireson CR, Jones DJ, Orr S, Coughtrie MW, Boocock DJ, Williams ML, Farmer PB, Steward WP, Gescher AJ: Metabolism of the cancer chemopreventive agent curcumin in human and rat intestine. Cancer Epidemiol Biomarkers Prev 2002, 11:105-111.

39. Hoehle SI, Pfeiffer E, Sólyom AM, Metzler M: Metabolism of curcuminoids in tissue slices and subcellular fractions from rat liver. J Agric Food Chem 2006, 54:756-764.

40. Kim H, Park BS, Lee KG, Choi CY, Jang SS, Kim YH, Lee SE: Effects of naturally occurring compounds on fibril formation and oxidative stress of $\beta$-amyloid. J Agric Food Chem 2005, 53:8537-8541.

41. Savonenko A, Xu GM, Melnikova T, Morton JL, Gonzales V, Wong MP, Price DL, Tang F, Markowska AL, Borchelt DR: Episodic-like memory deficits in the APPswe/PS1dE9 mouse model of Alzheimer's disease: relationships to $\beta$-amyloid deposition and neurotransmitter abnormalities. Neurobiol Dis 2005, 18:602-617.

42. Munro IC, Newberne PM, Young VR, Bär A: Safety assessment of Ycyclodextrin. Regul Toxicol Pharmacol 2004, 39(Suppl 1):S3-13.

43. Garcia-Alloza M, Robbins EM, Zhang-Nunes SX, Purcell SM, Betensky RA, Raju S, Prada C, Greenberg SM, Bacskai BJ, Frosch MP: Characterization of amyloid deposition in the APPswe/PS1dE9 mouse model of Alzheimer disease. Neurobiol Dis 2006, 24:516-524.

44. Belkacemi A, Doggui S, Dao L, Ramassamy C: Challenges associated with curcumin therapy in Alzheimer disease. Expert Rev Mol Med 2011, 13:e34.

45. Perkins S, Verschoyle RD, Hill K, Parveen I, Threadgill MD, Sharma RA, Williams ML, Steward WP, Gescher AJ: Chemopreventive efficacy and pharmacokinetics of curcumin in the $\mathrm{min} /+$ mouse, a model of familial adenomatous polyposis. Cancer Epidemiol Biomarkers Prev 2002, 11:535-540.

46. Ma Z, Shayeganpour A, Brocks DR, Lavasanifar A, Samuel J: Highperformance liquid chromatography analysis of curcumin in rat plasma: application to pharmacokinetics of polymeric micellar formulation of curcumin. Biomed Chromatogr 2007, 21:546-552.

47. Pan MH, Huang TM, Lin JK: Biotransformation of curcumin through reduction and glucuronidation in mice. Drug Metab Dispos 1999, 27:486-494.

48. Ireson C, Orr S, Jones DJ, Verschoyle R, Lim CK, Luo JL, Howells L, Plummer S, Jukes R, Williams M, Steward WP, Gescher A: Characterization of metabolites of the chemopreventive agent curcumin in human and rat hepatocytes and in the rat in vivo, and evaluation of their ability to inhibit phorbol ester-induced prostaglandin E2 production. Cancer Res 2001, 61:1058-1064.

49. Holder GM, Plummer JL, Ryan AJ: The metabolism and excretion of curcumin (1,7-bis-(4-hydroxy-3-methoxyphenyl)-1,6-heptadiene-3,5dione) in the rat. Xenobiotica 1978, 8:761-768.

50. Asai A, Miyazawa T: Occurrence of orally administered curcuminoid as glucuronide and glucuronide/sulfate conjugates in rat plasma. Life SCi 2000, 67:2785-2793.

51. Marczylo TH, Steward WP, Gescher AJ: Rapid analysis of curcumin and curcumin metabolites in rat biomatrices using a novel ultraperformance liquid chromatography (UPLC) method. J Agric Food Chem 2009, 57:797-803.

doi:10.1186/alzrt170

Cite this article as: Quitschke et al: The effect of cyclodextrin-solubilized curcuminoids on amyloid plaques in Alzheimer transgenic mice: brain uptake and metabolism after intravenous and subcutaneous injection. Alzheimer's Research \& Therapy 2013 5:16. 